\section{Our Winters in Relation to Brückner's Cycle.}

IT was said in Bacon's time that every thirty-five years "the same kind of suite of years and weathers comes about again" (see his essay "Of Vicissitude of Things"), and the important researches of Brückner on this subject are now receiving considerable attention.

The value, 35 years, as used by Brückner, is, of course, an average. The interval from centre to centre of his cold and wet periods (or the opposite) is sometimes as much as 40 , sometimes as little as 30 . It has been noted, further, that 35 is very nearly three times the sun-spot cycle of II.I years.

Now if we look into the variation of certain weatherelements at Greenwich since 1841 , it may, I think, be truly said to-day that the same kind of weather has come round again after about 33 years. Let us take $\epsilon . g$. our winter seasons as measured by the total number of frost days from September to May.

In the upper curve of the diagram herewith, each year point represents the sum of frost days in five winters so understood; the first (1844) for winters ending $1842-46$, the second, winters ending $1843-47$, and so on.

Similarly, in the lower curve, each point represents a five-winter group, but thirty-three years later, commencing

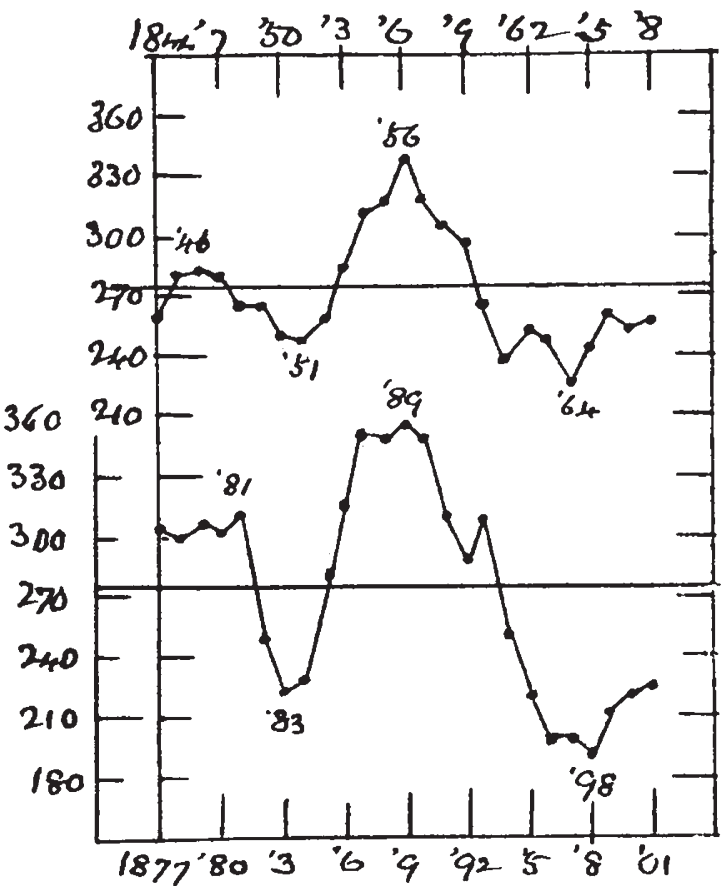

Curve showing the variation of frost days from five.jear sums for the periud $1842-1902$.

with 1877, and ending with 190I (which includes last winter).

There is obviously a general correspondence between these curves; high values in one matched with high values in the other, and low with low. Twenty-five pairs of values being thus compared, there are only four in which the members of the pair are on opposite sides of the average line (273).

Again, we have, in general,

\begin{tabular}{|c|c|c|c|c|}
\hline Diminishing cold & $\ldots$ & $\ldots$ & $\ldots$ & $1 \delta_{46} 6-51$ ( 5 years) \\
\hline Increasing cold & $\cdots$ & $\cdots$ & $\cdots$ & $\begin{array}{l}1851-5615 \\
1883-8016\end{array}$ \\
\hline Diminishing co'd & $\ldots$ & & $\ldots$ & $\begin{array}{l}1856-6418 \\
1889-98(9\end{array}$ \\
\hline
\end{tabular}

From these last dates there is a rise.

The earlier curve might thus be considered a kind of programme for the series of winters commencing 33 years after the first. It will be interesting, I think, to sed how far it continues to be so in the future.

The winters about 1856 and 1889 appear to have been eonspicuously cold times. We might, perhaps, anticipate another such time in the early 'twenties, the curve not rising so high between, though, of course, individual winters might be very severe. This seems to be suggested by the course of the curve after 1868 , but the cotre spondence may perhaps fail.

Other examples of such recurrence, corresponding more or jess closely, might be given. The long record of Rothesay rainfall (from 1800 ) will be found worth treatment in this way: conspicuously dry times occur about 1822, I 8,5 , and 1887 , and the smoothed curve from 1835 to date may be said to repeat in its main features that from 1802 to .1867 . Alex. B. MacDowall.

\section{An Ant Rubbed by a Lizard.}

THE following account of the robbery from an ant by a lizard may interest some of your readers.

While walking along the main road on the outskirts of Bordighera yesterday morning, I noticed a strange-looking insect moving across it in a peculiar way. On getting nearer, I saw that what had attracted my notice was a black ant-about an inch long with brown wings-dragging a cricket bigger than itself. It held the cricket by the head, and as the ant moved backwards it drew the cricket towards it. While doing so it entered the shadow cast by my umbrella, and instantly released its hold and got out of the shadow, but finding there was no danger it returned and seized its prey again by the head, and recommenced its backward movement. A low wall ran alongside the road, and when the ant got within six feet of it a common brown lizard appeared on the top of the wall and evidently soon caught sight of the ant, for it ran quickly down the wall and to within two feet of it, when it crouched for a second or two like a cat ready to spring, and then charged the ant, apparently butting the cricket free with its head. Before the ant could regain its hold the lizard seized the cricket in its mouth, and darted up the wall in the direction from which it originally appeared on the scene, leaving the ar.t running round and round, moving its wings in an agitated manner, vainly searching for its lost prey.

Villa Mona, Bordighera, September r.

$$
\text { J. W. Stack. }
$$

\section{A NEW MECHANICAL THEORY OF THE AET HER.'}

THIS memoir was communicated to the Royal Society in February, I902, and has now been issued in the dual form of a contribution to the Philosophical Transactions and a volume of Prof. Osborne Reynolds's collected papers.

It may safely be described as one of the most remarkable attempts that have been made of recent years t.) formulate a dynamical system capable of accounting for all physical phenomena at present known. A theory such as is here set forth may not improbably play the same part in modern science that was assumed by the atomic theory and the kinetic theory of gases in the science of the time when these theories were propounded.

If we suppose the ultimate particles--Prof. Reynolds calls them "grains"--constituting the material universe to be either spheres, or what comes to the same thing, point atoms behaving in the same manner as uniform smooth spheres, then it is impossible to assume these grains to be of equal size and distributed at random through space without assuming them (as in the kinetic theory of gases) to be in motion among themselves. On the other hand, a medium in which the motion of the different grains among themselves partakes of the nature of diffusion does not lend 1 "The Sub.Mechanics of the Universe." By O.borne Reynolds, M.A., F.R.S., LL.D., M.Inst.C.E. Pp. xvii + 256 . (Cambridge Un versity Press:
1os. 6 . net.

No. 1773 , voL. 68] 
itself kindly to the explanation of such phenomena as the propagation of transverse waves. The medium considered in the present investigation is assumed to consist of uniform spherical grains which are so close together as to prevent diffusion, and when in a state of " normal piling" the centre of each grain is supposed to be equidistant from those of twelve neighbouring grains, this being the distribution corresponding to minimum volume, and the system " constituting to a first approximation an elastic medium with six axes of elasticity symmetrically placed." [It may be pointed out before proceeding further that there is more than one way of piling balls so that each ball is in contact with twelve neighbouring ones and the total volume is a minimum.]

The grains are supposed to be capable of limited relative motion, and local inequalities may exist due to the presence or absence of a number of grains above or below that necessary for normal piling. In such cases singular surfaces are formed between the grains in unstrained and those in strained piling. The author finds that the local negative inequalities produced by the absence of grains present the ordinary properties of matter. They are free to move through the medium without resistance, the grains streaming freely through their singular surfaces, and they attract one another according to the law of the inverse square. The density of matter is thus negative, taking that of the medium to be positive, and if the density of water be taken as -1 , the author finds that of the medium to be $10^{4}$. The diameter of the grains in C.G.S. units is $5.534 \times 10^{-18}$, their mean path is $8.612 \times 10^{-28}$, their mean relative velocity is $6.777 \times 10$, the mean pressure is $1.172 \times 10^{14}$, the rate of propagation of the transverse wave is $3.004 \times 10^{10}$, and the rate of degradation of the transverse wave is such that it would require 56 million years to reduce the total energy in the ratio of I to $e^{2}$. The absorption thus produced is of such a magnitude as to account for the blackness of the sky on a clear night compatibly with the absence of any measurable absorption of light by the ether. On the other hand, the absence of any evidence of normal waves until quite recently is accounted for by the conclusion that the rate of degradation of the normal wave would reduce its energy to about one-eighth in $3.923 \times 10^{-6}$ of a second, or before it had traversed 2200 metres. In addition to positive and negative inequalities of which the latter correspond to matter the existence is assumed of "complex inequalities" due to the displacement of grains from one position to another, and a comparison of the attractions of such inequalities with those due to the inequalities representing matter is in complete accordance with the known smallness of gravitative as compared with electric action.

The theory accounts for the refraction, dispersion, polarisation by reflection, metallic reflection and aberration of light.

The analytical investigation is based on the general equations of motion and conservation of any entity (Section ii.), these equations being generalisations of the well-known equations of continuity of hydrodynamical and allied systems; the formation of the equations of motion in a purely mechanical medium (Section iii.), the separation of the motion into its components of "mean" and relative motion (Sections iv.-vii.), the extension of the kinetic theory to granular media (Sections viii.-x.), and an elaborate analysis of the changes taking place in the angular inequalities, the momentum and energy, the mean and relative systems, and the mean inequalities and their motions (Sections xi.-xiv.). It should be observed that ths present theory involves the assumption that positively electrified bodies do not repel each other, and for this the author gives arguments in $\S 226$. In the final section (xv.) the numerical values of the quantities which define the condition of the granular medium, as stated above, are deduced from the results of physical experience.

The mathematical reasoning is very difficult, in some places almost impossible, to follow, owing to the large number of doubtful points or inaccuracies in the equations. Even if the fundamental conclusions should prove to be correct, there are many points in the argument which are at present obscure, and require to be cleared up. 'To take a few examples, in equation (4), $p_{1}, \ldots$, a new symbol $r$ is introduced without any explanation, and the dual use of $\delta$ is very confusing. Having used $\delta S$ to denote a volume element, and $\delta$ s a surface element on this page, the author suddenly changes from $\delta S$ to $\delta s$ in the first of equations (20) on $\mathrm{p} 16$, and to $d s$ in the second and third, although he refers to equation (2) of $p$. 10 , which involves $\delta S$. On $\ddot{p} . r_{3}$ in equation ( $\left.r_{3}\right)$, the differential is omitted after the treble sign of integration; also in (16) one of the expressions under the sign of summation is multiplied by the differential element $d S$, while the other is not; in the former equation the reader will naturally supply the missing $d x d y d z$, but the meaning of the latter equation is obscure. Again, turning to p. I05, we find that $\S 116$ refers to "The mean velocities of pairs having relative velocities $\sqrt{2}_{2} \mathrm{~V}_{1}^{\prime}$ and $\mathrm{V}_{1}^{\prime} / \sqrt{2}_{2}$," while in $\S 120$ we read, "Since the mean velocities of pairs of grains having relative velocity $\sqrt{ }_{2} 2 \mathrm{~V}_{1}^{\prime}$ is $V_{1} / \sqrt{ }_{2} \ldots .$. In $\S$ II $_{7}$, "All directions of mean velocity of a pair are equally probable whatever the direction of the mean velocity." On p. 120, equation (181), it is not easy to see how, if $\mathrm{N}$ be equal to the number of grains in unit volume, the square root of $\mathrm{N}$ should be equal to $\mathrm{N} d x d y d z$ multiplied by a certain function of the coordinates, nor how by integrating the equation with respect to $y$ and $z$ the square root of $\mathrm{N}$ now becomes equal to $\mathrm{N}$ multiplied by another function multiplied by the linear differential $d x$. In ordinary circumstances there is no useful purpose served in filling a review with a list of errata which any reader could easily correct for himself. But the present investigation would be difficult to follow even under the most favourable conditions, and the presence of so many formulæ and statements which cannot possibly be correct as they stand renders the task well nigh hopeless.

An objection of an entirely different character applies to the sections in which Maxwell's law of distribution of velocity components and partition of energy is extended to a medium of closely packed spheres such as that considered by Prof. Reynolds. A great deal has been written as to the validity of Maxwell's law, and of the fundamental assumptions involved in the proofs of it. The general opinion on which all mathematical physicists are pretty well agreed is that the law holds good to a first approximation in gaseous media the molecules of which are not too closely crowded together; but one method of proof after another has on closer examination been found to involve some assumption or other which usually breaks down in the case even of a dense gas. Moreover, Mr. Burbury has gone so far as to establish a different formula for the law of distribution in dense gases. To assume the law to hold good in the extreme case of a medium the ultimate particles of which are permanently interlocked must be regarded, failing other evidence than that given by Maxwell, as a very doubtful step.

A number of interesting questions suggest themselves for the consideration of physicists, such as the ultimate distribution of energy between the grains and molecules, the determination of the temperature of cosmic space as defined by the mean kinetic energy of the grains, the influence of the absorption of the medium, however small, on the progress of cosmic 
events, the existence of directional properties of the ether determined by the regular arrangement of the grains, and the finity or infinity of extent of the medium. It may be confidently. anticipated that Prof. Osborne Reynolḋs's granular medium will play an important part in the physics of the future. It is, however, to be hoped that the subject will receive careful and critical study in the hands of numerous mathematical physicists, and that it will not be left for the experimenter and philosopher blindly to accept Prof. Revnolds's doctrines as the basis of speculations about things which they do not understand. The practice of assuming statements to be true because Maxwell made them has been too prevalent in the past, and there is not very much difference between those who adopt this attitude and writers who publish papers at their own expense to show that the earth is not round or that gravitation does not exist. The dogmatic statements of the former class of philosopher often afford plenty of material for the abusive attacks of the latter.

$$
\text { G. H. BRYAN. }
$$

\section{THE EFFECT OF EDUCATION AND} LEGISLATION ON TRADE.

$\mathrm{N}$ his second presidential address to the Society of Chemical Industry at its annual meeting held in Bradford, Mr. Levinstein again addressed himself to the subject of education. He thinks that almost too much importance has been attached to education as being the only factor which has caused the industrial progress and superiority, in certain classes of merchandise, of Germany in comparison with this country. Attention is therefore directed to other considerations which he considers have also to be taken into account, such as the unification of the various German States after the Franco-German war, which, of course, gave an internal free trade to the German nation, the nationalisation of the railways and canals, and the protective patent laws.

He then refers to America, which he does not consider to be a better educated country than our own. Naturally the new Education Act of 1902 comes under review. Mr. Levinstein is doubtful, as are many others, if the Bill will advance secondary education, because the number of persons appointed to the councils who represent secondary education is excecdingly small. No remark is made upon another aspect of the case, namely, that a great many of those appointed know practically nothing about primary, and still less about secondary education. In some cases which have come before our notice, persons of little education (beyond their own inflated opinion of themselves), but desirous of local fame, and having plenty of " push," have brought themselves forward and been elected, while those who really are educated, and know what education means, have been passed by.

The raising of secondary education to a really high and uniform standard will be extremely costly. But the expenditure on primary education, according to the provisions of the new Act, will absorb such a large amount of the ratepayers' money that they will be disinclined to incur further expenditure in order to make it really efficient. No student can enter a German technical college without passing an extremely searching and thorough examination. In Great Britain the total number of students, from fifteen years and upwards, taking complete day technological courses is 3873 ; probably not more than ro per cent. could pass the entrance examination of Charlottenburg.

$\Lambda$ s an illustration of what Manchester is doing in the way of technical training, Mr. Levinstein gives an account of "the department for preparing, bleaching, dyeing, printing, mercerising and finishing textiles, together with the manufacture of paper." There is no dabbling here with manufacture in a test tube, such as we see in some of our educational institutes. The department is lodged in a separate building apart from the school of technology. It is fitted with the latest and most up-to-date machinery, taken from this country and abroad. As all the machinery is driven by separate motors, there will be no difficulty in replacing it, as it becomes out of date and obsolete, by means of newer and more modern machinery.

In this country we excel in the production of firstclass varn and cloth, made from first-class raw material. These goods will always fetch a good price. But within the last quarter of a century a demand has sprung up for cheap imitations, made from inferior materials, but which must have the external appearance of the first-class article. It is in the weighting of silk, the intermixing of fibres and the manufacture of imitation velvets that the foreigner excels. But the demand is enormous, and if we would hold our own in the markets of the world, we must learn how to manufacture these cheap goods. The British manufacturer must learn to adapt himself to the times and to the tastes and wishes of the consumer.

Manufacturers have often refused to employ chemists, except as "testing machines," because the chemist is so often only a theorist, sometimes not ever. that, and understands absolutely nothing about machinery. This excuse will, however, soon be no longer tenable. Students who have passed through the department just mentioned at the Manchester Technical School should be fully qualified to take a position not only in dye, bleach, print, mercerising, or fitushing works, but also in paper mills. They will have not only a knowledge of chemistry, but also of machinery. It is a pity that technical institutes do not make it compulsory for those who intend to become works chemists to include in the syllabus a ccurse in engineering, both practical and theoretical.

Referring to the "Patent Law Amendment Act," Mr. Levinstein has great hopes that satisfactory results will accrue to our manufacturers. The chief clause in the Act, and one for which British manufacturers have been agitating for many years, is that which deals with the granting of licences. Hitherto the foreigner could patent anything he chose, manufacture it abroad, and "dump" it down here, without his being under any obligation to manufacture it on British soil. And it was a matter of great difficulty to compel him to grant a licence to a British firm to manufacture the goods. Under the new Act, if he does not manufacture in this country, he can be compelled to grant a licence for the manufacture of the product, or failing this his patent may be declared void.

It is only after more than twenty years of agitation that this Act has been passed. Mr. Levinstein reviews the pioneering work which had to be done before the inertia of the Board of Trade was overcome.

Finally, the difficult and vexed question of foreign tariffs is dealt with. Mr. Levinstein considers that the reasons we have not made greater headway, so far as our export trade is concerned, are:-our education has been at fault, our patent laws were bad, and foreign tariffs have often been prohibitive; and we would add the want of adaptability of some of our manfacturers. The Government is also exceedingly slack in making known to our traders, at the earliest moment possible, changes in foreign tariffs. Interested Continental traders learn at once, through their Minister of Commerce, not only changes which have taken place, but changes which are contemplated. But the wheels of our Government, in respect to information which may 